\title{
OTIOTOMICS
}

Revista de economía, empresa y sociedad

Dossier «Economía social y solidaria: experiencias y retos»

\section{La economía social y solidaria en el siglo xxr: un concepto en evolución. Cooperativas, B corporations y economía del bien común}

\section{Vanessa Campos-i-Climent}

Profesora ayudante doctora del Departamento de Dirección de Empresas. Universitat de València

RESUMEN Todas las formas de organización social que han existido lo largo de la historia de la humanidad han satisfecho las necesidades de sus integrantes de diferente manera. Es decir, han dado respuesta a las tres cuestiones esenciales que se plantea la ciencia económica: «¿qué producir?», «¿cómo producir?» y «¿para quién producir?». Pero el único rasgo que han compartido todas ellas es que la unidad básica de producción de los bienes y servicios es la empresa. Por tanto, la manera en que se toman las decisiones dentro de las empresas, el juego de poder que determina qué intereses se priorizan en cada momento, resulta clave para entender cómo se da respuesta a estas tres cuestiones antes mencionadas. Cuando se plantea la necesidad de un cambio hacia una economía que ponga las personas en primer lugar, lo que nos tendríamos que plantear es si hay otra manera de hacer empresa. Con ese objetivo, en este artículo se hace una revisión de las formas empresariales llamadas "críticas", que se basan en el cuestionamiento del principio capitalista según el cual el principal objetivo de las empresas tiene que ser generar cuánto más dinero mejor. Revisaremos y compararemos qué son y cómo surgen estas formas empresariales críticas: desde las cooperativas, pasando por las sociedades laborales, las $B$ Corp y la economía del bien común.

PALABRAS CLAVE economía social y solidaria; cooperativas; sociedades laborales; B Corp; economía del bien común

Social and solidarity economy in the $21^{\text {st }}$ century, a concept in evolution: co-operatives, B Corporations and Economy for the common good

ABSTRACT All forms of social organization that have existed throughout human history have satisfied human needs in diferent ways. That is, they have given different answers to the three basic questions considered by Economics: "what to produce?", "how to produce it?" and "for whom to produce it?" The only trait that all forms of social organization have in common is their consideration of enterprises as the basic unit of production for goods and services. 
Therefore, the way decisions are made in firms - the power game forever determining which interests are given priority over others - is key to understanding how the three above-mentioned questions are resolved. As such, faced with various proposals calling for an enconomy that prioritizes people over capital, we need to consider alternative ways in which business can operate.

In light of the above, this paper reviews the wide range of business models that criticize or question the principal of investor-owned firms, ie organizations whose main goal is not to make a profit whatever the cost. The paper provides an overview and comparison detailing the origins of such business models, which include co-operatives, labour-managed firms, B Corps, and Economy for the common good.

KEYWORDS social and solidarity economy; co-operatives; labour-managed firms; B Corp; Economy for the common good

\section{Introducción}

El concepto de economía social nace en la Inglaterra del siglo xIX, como respuesta de los obreros de la industria textil a sus pésimas condiciones de vida. Las primeras manifestaciones de la economía social se dan en Rochdale en 1844, donde los conocidos como «Pioneros de Rochdale» formaron una cooperativa de consumidores que les permitía abastecerse de víveres y menaje del hogar a unos precios significativamente inferiores a los del mercado. Consistía en una respuesta colectiva articulada desde la sociedad civil para dar solución a los problemas reales de dicha sociedad; se trataba, pues, del planteamiento de un modelo económico alternativo a la economía capitalista y a la economía marxista.

Mucho más adelante, durante la década de los años sesenta del siglo xx, en el Estado español empezó a gestarse el modelo de las sociedades laborales. Este modelo de empresa implica una forma jurídica que combina la lógica cooperativista con la lógica de una sociedad capitalista y se desarrolló de manera considerable durante los años setenta, hasta conseguir por primera vez un reconocimiento legal con la Ley de sociedades anónimas laborales, de 25 de abril de 1986, y la posterior Ley de sociedades laborales, de 24 de marzo del 1997.

Más reciente es la realidad de las empresas sociales. Sin forma jurídica determinada, para ser clasificadas como tal, tienen que ser organizaciones cuyos objetivos tengan una doble naturaleza: económico-financiera y social. Las empresas sociales son muy numerosas y están muy desarrolladas en los países anglosajones y en el norte y centro de Europa. En el caso de España, las más numerosas son las empresas de inserción, cuyo principal objetivo es introducir en el mercado laboral a personas en riesgo de exclusión social a causa del trabajo.

En 2006, un grupo de empresarios y profesionales de Estados Unidos crearon B Lab, una organización sin ánimo de lucro cuya finalidad era desarrollar un sistema de certificación que evalúe el impacto de las empresas en la sociedad. Así definieron el concepto de $B$ Corporation (o $B$ Corp), que ya no está planteado en función de la forma jurídica de la empresa, sino de su comportamiento de cara a la sociedad y el medio ambiente.

En Europa, el filólogo Christian Felber publicó el libro Economía del bien común (2010), en el que señalaba, como ya hicieron los pioneros de Rochdale a mediados de siglo xIX, la necesidad de que la economía pusiese a las personas en primer término. Felber proponía que las empresas y el resto de organizaciones elaborasen un balance del bien común para medir su impacto en la sociedad.

Con el objetivo de analizar y comparar estas realidades, el presente artículo se estructura en tres secciones: en la primera se conceptualizan las formas empresariales más tradicionales que conforman la realidad de la economía social y solidaria; en la segunda se describen y analizan las aportaciones más recientes de las $B$ Corps y la economía del bien común, y finalmente, en la tercera se hace un análisis comparativo de los diferentes modelos y se señalan las principales conclusiones. 


\section{Las empresas tradicionales de la economía social y solidaria: cooperativas y sociedades laborales}

Como se ha señalado en la introducción, las empresas de la economía social y solidaria más numerosas y con una trayectoria más larga y consolidada en el tiempo son las cooperativas. La Alianza Cooperativa Internacional (ACl, 1995) define una cooperativa como una asociación autónoma de personas que se unen voluntariamente para conseguir sus aspiraciones económicas, sociales y culturales a través de una empresa de propiedad conjunta y gobierno democrático.

En las empresas cooperativas, la toma de decisiones está basada en los valores de ayuda mutua, corresponsabilidad, democracia, igualdad, justicia y solidaridad. De estos valores emanan los siete principios cooperativos que sirven de guía para que las empresas cooperativas lleven sus principios a la práctica ( $\mathrm{ACl}, 1995)$ :

1. Adhesión voluntaria y abierta.

2. Gestión democrática.

3. Derecho de los socios a participar de los resultados generados por la cooperativa.

4. Autonomía e independencia de los poderes políticos.

5. Educación, formación e información para los socios, directivos, trabajadores y representantes.

6. Cooperación entre cooperativas.

7. Preocupación e implicación por la comunidad.

Para cuantificar lo que suponen las empresas cooperativas en la economía mundial, habría que señalar que, de acuerdo con datos que recoger el World Co-operative monitor 2014, publicado conjuntamente por el ACl y el Instituto Europeo de Investigación en Cooperativas y Empresas Sociales (EURICSE), en 2014 las cooperativas facturaron en todo el mundo 2,2 trillones de dólares y ocupaban a 250 millones de personas. La contribución de las empresas cooperativas a la generación de ocupación es más que relevante, tanto desde el punto de vista cuantitativo como cualitativo, suponiendo el 12\% de la población ocupada en los países del G-20.

Por lo que respecta al número de socios y al de empresas cooperativas, Estados Unidos es el país del mundo donde más personas son socias de cooperativas: 256 millones de socios y cerca de 30.000 cooperativas. En Québec (Canadá), cerca del 70\% de la población son socios de alguna cooperativa. En Asia, los países donde las cooperativas tienen más relevancia son la India y Japón. En el primero hay 93,7 millones de personas que son socias de alguna cooperativa, mientras que en Japón son 77 los millones de socios, con un dato muy destacable: el 91\% de los agricultores japoneses están asociados en cooperativas. En Oceanía destaca el caso de Nueva Zelanda, donde el 3\% del PIB lo producen las cooperativas, copando hasta el 95\% de las exportaciones de productos lácteos (tanto frescos cómo elaborados). Finalmente, en Europa el país donde más peso tienen las cooperativas es Italia, con 22,5 millones de socios.

En cuanto al España, la Ley 5/2011, de 29 de marzo, define la economía social como el conjunto de actividades económicas y empresariales que, en el ámbito privado, llevan a cabo aquellas entidades que, en conformidad con los llamados (por la propia ley) principios orientadores, persiguen el interés general económico o social o ambos. La ley también enumera cuáles son, en el Estado español, las empresas de la economía social.

Dentro de las llamadas for profit organizations, encontraríamos principalmente, las cooperativas y sociedades laborales. Pero también estarían: las mutualidades, los centros especiales de ocupación y las empresas de inserción. Por otro lado, dentro de las llamadas not for profit organizations, encontramos las asociaciones, las fundaciones y las cofradías de pescadores.

De acuerdo con datos de la Confederación Empresarial Española de la Economía Social (CEPES), a finales de 2015 había en España 42.929 empresas de la economía social, que generaron el 10\% del PIB. De estas, $20.258(47,19 \%)$ eran cooperativas y 10.828 sociedades laborales $(25,22 \%)$. Si sumamos ambas, cooperativas y sociedades laborales suponen 31.086 empresas, un 74,41\% (casi dos terceras partes) del total de empresas de la economía social. 
En cuanto a la ocupación generada, y de acuerdo con las estadísticas del Ministerio de Ocupación y Seguridad Social, las cooperativas daban ocupación directa a 277.390 personas a finales de 2015. Las sociedades laborales ocupaban de manera directa a 63.536 personas. Se estima que el total de ocupación directa e indirecta generada por las empresas de la economía social en 2015 era de 2.219 .733 puestos de trabajo en todo el estado. En total, las empresas de la economía social generaron en 2015 el 12,5\% de la ocupación del estado, con un $80 \%$ de contratación indefinida y un $47 \%$ de ocupación de personas menores de 40 años.

Por territorios, Cataluña es la comunidad donde las cooperativas son más numerosas: 4.138 de las 20.258 $(20,43 \%)$ cooperativas son catalanas. Le siguen Andalucía, con 3.973 (19,61\%), y el País Valenciano, con 2.396 $(11,82 \%)$.

Figura 1. Número de cooperativas en 2015

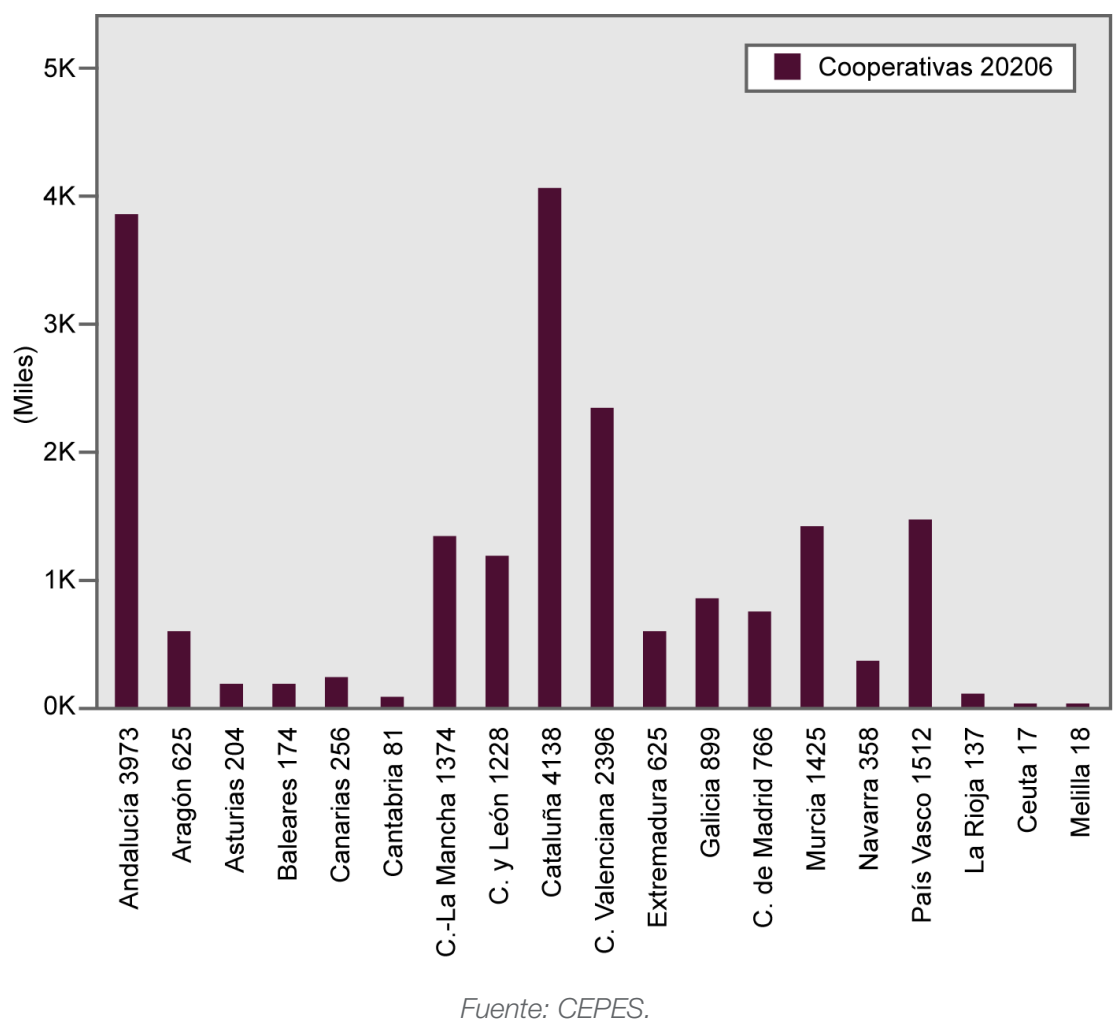

La distribución de la ocupación directa generada por las cooperativas en los diferentes territorios durante el 2015, de acuerdo con las estadísticas del Ministerio de Ocupación y Seguridad Social, sitúa en primer lugar al País Vasco, donde las cooperativas ocupan a 54.337 de las 277.390 personas de todo el estado (19,58\%). En segundo lugar, las cooperativas andaluzas ocupan a 43.897 personas (15,82\%), seguidas por las cooperativas catalanas, con 42.232 ocupados (15,22\%), y las valencianas, con 37.079 ocupados (13,37\%). 
Figura 2. Ocupación en cooperativas en 2015

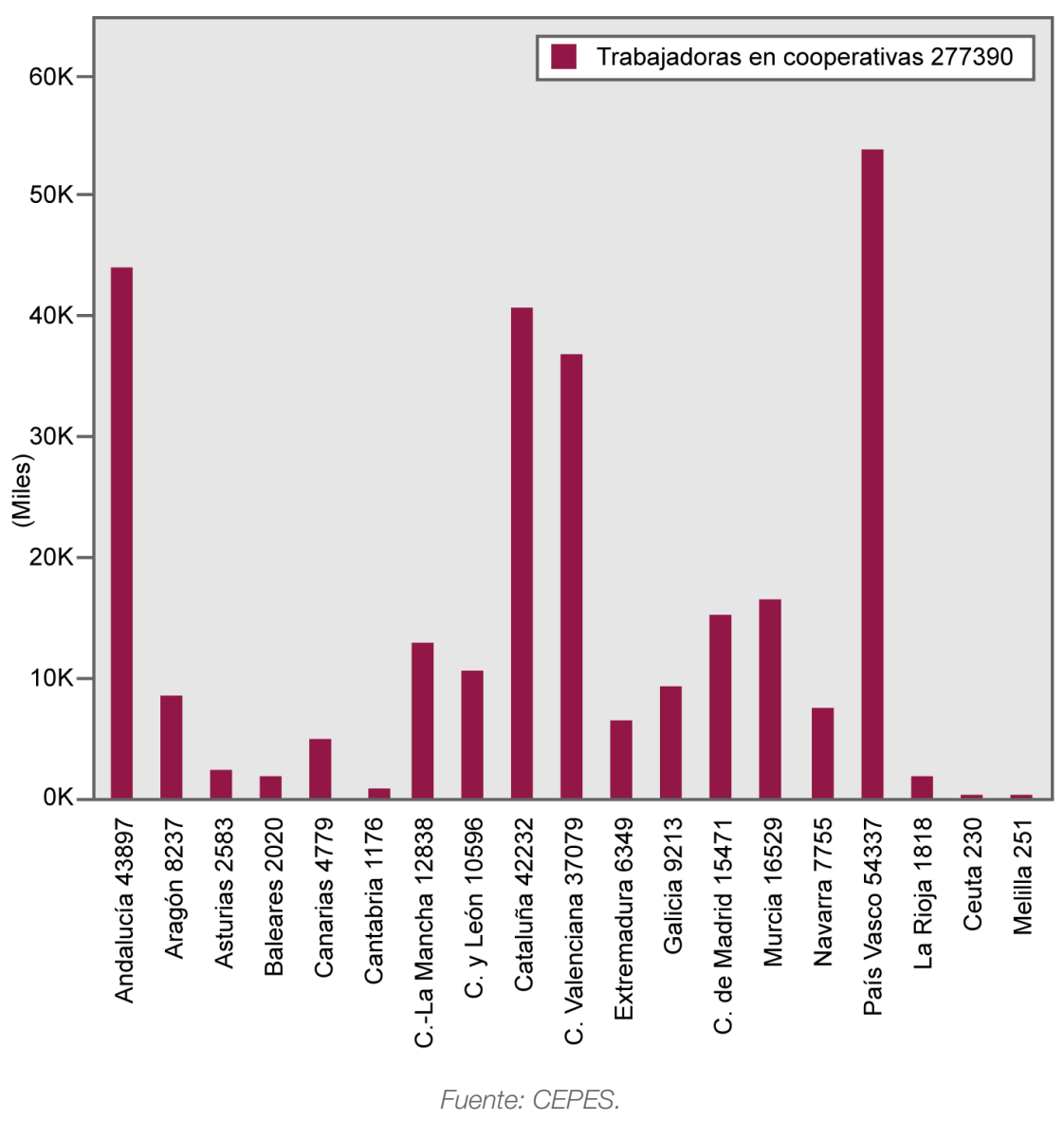

El otro tipo de empresa de la economía social que resulta más relevante dentro de nuestra realidad son las sociedades laborales (sociedades anónimas laborales -SAL-y sociedades limitadas laborales -SLL-). Estas empresas tienen los mismos requerimientos de capital social mínimo que las casi-homónimas sociedades capitalistas, pero se diferencian de estas por el hecho que al menos el $51 \%$ del capital es propiedad de los socios-trabajadores (aunque ningún socio-trabajador puede poseer más del 30\% del capital social), pudiendo haber socios capitalistas, mientras que los socios-trabajadores pueden optar entre cotizar en el régimen general de la Seguridad Social o en el de autónomos. Las sociedades laborales tuvieron una cierta relevancia dentro del mundo de la empresa en las décadas de los años setenta y ochenta, puesto que permitieron que muchas sociedades capitalistas (principalmente industriales) en crisis sobrevivieran mediante su transformación en sociedades laborales. De este modo, las sociedades laborales y las cooperativas de trabajo asociado han sido tradicionalmente los modelos de empresa que han permitido salvar puestos de trabajo en épocas de crisis, dado que han constituido las formas jurídicas que permitieron la existencia de las llamadas empresas recuperadas por sus trabajadores. Sin embargo, fuera del Estado español las sociedades laborales no han tenido el mismo desarrollo. De hecho, en muchos países del mundo ni siquiera existen. 
Figura 3. Número de sociedades laborales en 2015

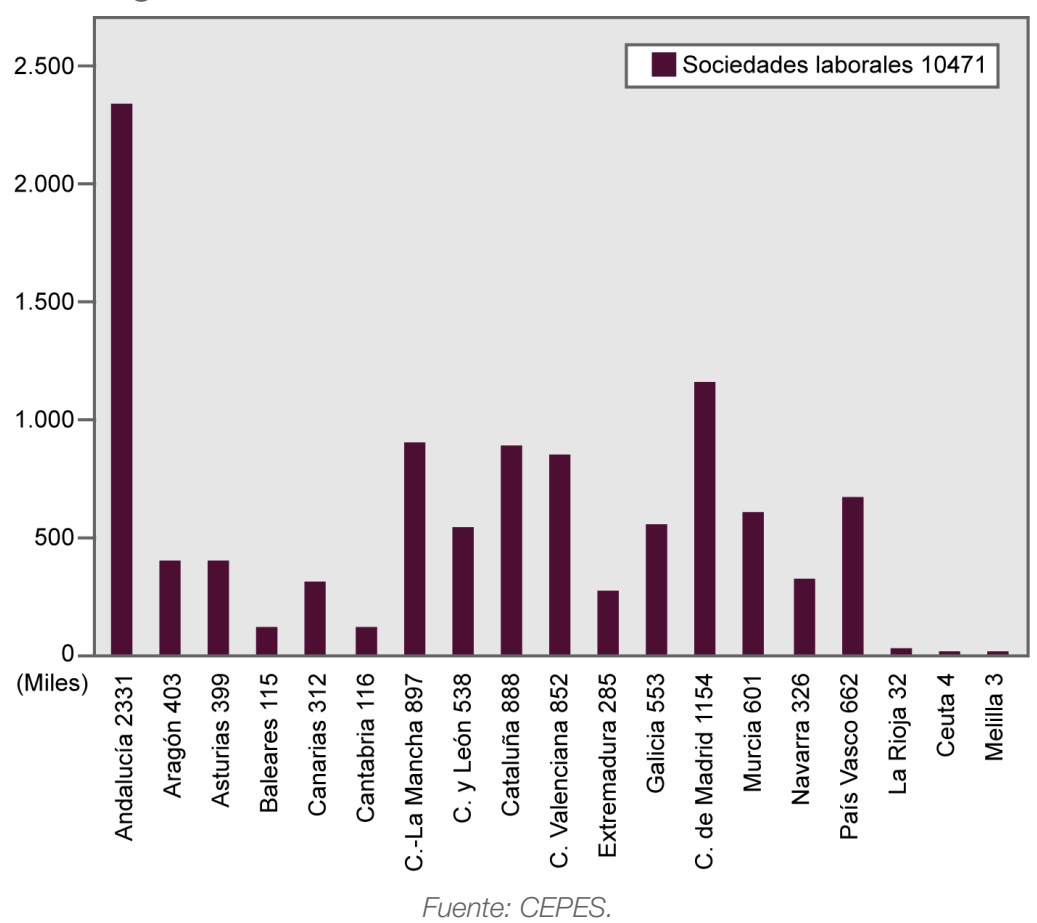

Desde el punto de vista cuantitativo, según datos del Ministerio de Ocupación y Seguridad Social, a finales de 2015 había en todo el estado 10.471 sociedades laborales, que daban trabajo a 64.491 personas. Por territorios, el lugar donde más sociedades laborales hay localizadas es Andalucía, con 2.331 empresas que ocupan a 12.811 personas, seguida por la Comunidad de Madrid, con 1.154 empresas que ocupan a 7.367 personas.

Figura 4. Ocupación en sociedades laborales en 2015

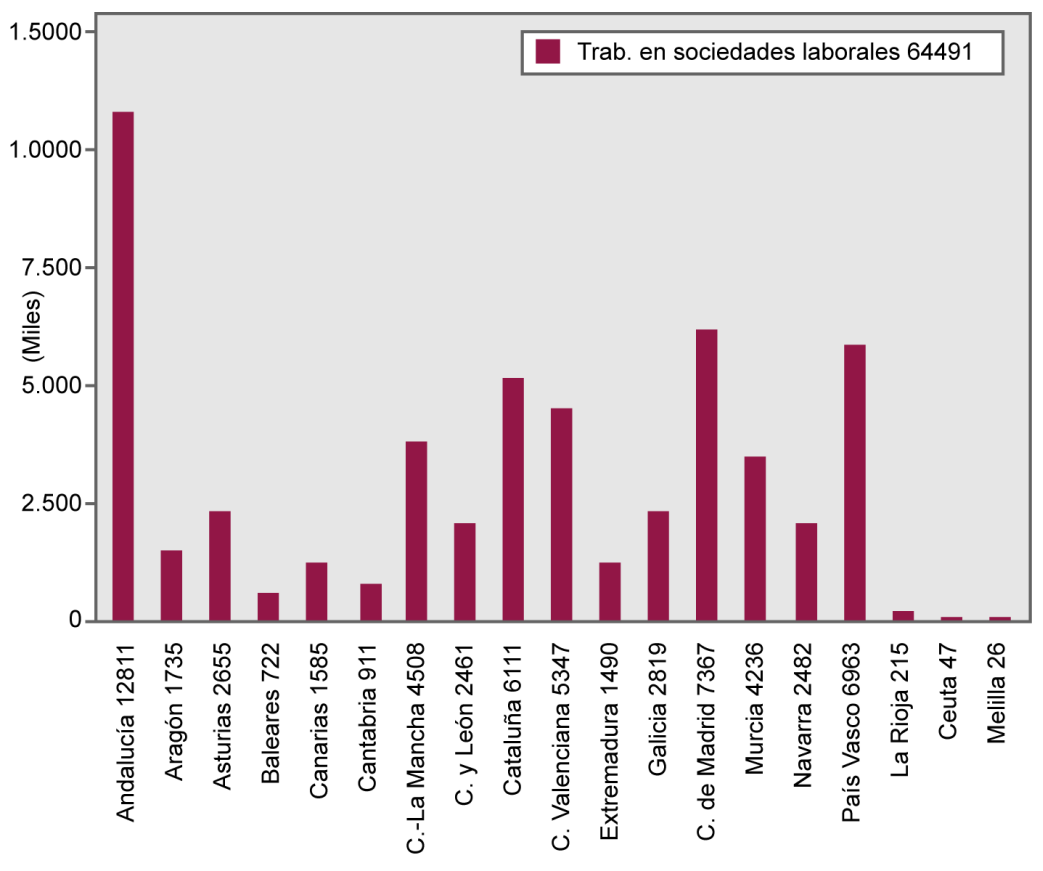

Fuente: CEPES. 


\section{La nueva realidad: aportaciones de las B Corp y la economía del bien común}

Poco antes de la primera gran crisis económica del siglo XXI, en el 2006, un grupo de empresarios, profesionales y académicos estadounidenses formaron el B Lab, una organización sin ánimo de lucro, con la convicción de que la principal finalidad de cualquier tipo de empresa (incluidas las capitalistas) no podía ser generar beneficios al precio que fuera, y que en el balance de las empresas con respecto a la sociedad y el territorio había que incluir también el impacto social y medioambiental para evaluar correctamente la sostenibilidad de las actividades empresariales. Se trata pues de un proyecto nacido y desarrollado colectivamente.

En Europa, tras la publicación en 2010 de la obra Economía del bien común, escrita por el filólogo austriaco Christian Felber, se pone en marcha un movimiento que aboga por que las empresas de cualquier tipo incorporen objetivos sociales y de sostenibilidad. El movimiento surge como respuesta a la crisis del 2008. En este caso, se trata de un proyecto nacido en el ámbito individual que influye en el colectivo, gracias al claro liderazgo de su impulsor.

A lo largo de este apartado expondremos cuáles son los rasgos más relevantes de los dos modelos, comparándolos entre sí, y qué aportación suponen respecto de las empresas tradicionales de la economía social (cooperativas y sociedades laborales).

\subsection{Las $B$ Corporations, una fuerza para el bien}

La visión que guía la definición de $B$ Corporation está basada en la idea de que las empresas y el mundo de los negocios pueden actuar como fuerza para hacer el bien en una economía globalizada. De este modo, las $B$ Corp tienen que ser capaces de crear beneficios (no solo económicos o financieros) para todos los stakeholders y no solo para los propietarios de la empresa.

Esta definición intenta trasladar de alguna manera la mayor parte de los principios y valores cooperativos a las empresas capitalistas. Freeman (2010) define los stakeholders como los grupos de personas o individuos que pueden afectar o verse afectados por el logro de los objetivos de la organización. Es por eso que tendrían que ser tenidos en cuenta a la hora de tomar decisiones por parte de las organizaciones (Bryson, 2004).

Por tanto, seguir este principio supone ampliar los grupos sociales que se han de tener en cuenta a la hora de tomar las decisiones en el seno de la empresa, no limitándolos solo a los grupos más próximos, como son los propietarios del capital y/o los trabajadores. Pero a diferencia de las cooperativas y las sociedades laborales, la capacidad de influencia ya no vendría solo dada por la propiedad, sino también por las propias relaciones de la empresa con los grupos del entorno; por ejemplo, un grupo ecologista local, aunque no formara parte de la propiedad de la empresa, podría llegar a llegar a tener mucha influencia.

La definición de $B$ Corp no va ligada a ninguna forma jurídica determinada; así, hay B Corps que son cooperativas, como Cabot Coop. (Vermont, EE. UU.), y otras que no lo son, como Ben \& Jerry's (Vermont, EE. UU.).

Desde B Lab, el grupo promotor de este movimiento, se impulsó un completo sistema de certificación que define qué variables hay que tener en cuenta, con sus correspondientes instrumentos de medida (indicadores), para evaluar el impacto social y medioambiental de la actividad empresarial. Se trata de un sistema que cumple con todos los estándares que requiere una auditoría y consiste en una adaptación del Balanced scorecard (Kapplan y Northon, 1996), donde variables e indicadores estarían interconectados permitiendo el análisis de la empresa como un sistema integrado. La primera empresa certificada como B Corp fue Bailo el 8 de junio de 2007.

La certificación $B$ Corp se creó con el fin de permitir una información transparente a consumidores, empresas y administraciones públicas en cuanto al comportamiento desde el punto de vista social y medioambiental de las empresas a la hora de adquirir sus productos. El 23 de abril de 2010, el estado de Maryland introdujo en la legislación algunas formas de tratamiento favorable a las empresas certificadas como B Corp por entender que estas contribuyen al bien común de la sociedad.

Actualmente hay 1.854 B Corporations en todo el mundo, de 150 países diferentes que operan en 130 sectores de actividad. 
En definitiva, el sistema de certificación creado por B Lab se ha convertido en una herramienta muy útil para que las empresas (con cualquier forma jurídica) incorporen principios y valores sociales y medioambientales, lo que supone incorporar en gran medida los principios y valores cooperativos.

\subsection{La economía del bien común}

Por su parte, la economía del bien común también intenta que las empresas capitalistas incorporen los principios y valores propios de la economía social. Cómo en el caso de las B Corp, también se basa en el enfoque de los stakeholders para diseñar un sistema de auditoría del bien común. Con esta auditoría se pretende evaluar el grado de desempeño de los criterios sociales. El instrumento en el que se basa es la llamada matriz del bien común, en la que se definen una serie de variables ligadas a diferentes stakeholders. Estas variables tienen una serie de indicadores asociados para medir su grado de cumplimiento. Actualmente, la matriz del bien común va por su quinta versión.

La matriz del bien común tiene en cuenta los siguientes valores: dignidad humana, solidaridad, sostenibilidad ecológica, justicia social y participación democrática, y transparencia. Y los analiza teniendo en cuenta el impacto en los siguientes stakeholders: proveedores, financiadores, empleados (incluyendo a los propietarios), clientes, productos, servicios, coempresas y ámbito social (región, soberanía, generaciones futuras, personas y naturaleza mundial). Además, hay algunos aspectos que pueden penalizar, es decir, restar puntos a la suma anteriormente conseguida.

A diferencia de la certificación de las B Corp, las variables e indicadores no funcionan como un sistema interconectado, sino que se trata de diferentes secciones que van sumando puntos. Otra diferencia importante es que mientras la economía del bien común intenta alcanzar la certificación de territorios, municipios, administraciones públicas y empresas privadas (todo tipo de organizaciones), el certificado B Corp solo atañe a organizaciones del sector privado, cuyo impacto social, no lo olvidemos, se mide de forma muy diferente al del sector público.

Otro rasgo diferenciador es que la economía del bien común es un concepto inspirado en realidades que ya existían, creado por una persona, Christian Felber, y desarrollado posteriormente de manera colectiva tomando como base el liderazgo de su creador. Tanto en el caso del concepto de economía social y solidaria como en el de $B$ Corp se trata de conceptos que se generan y desarrollan de manera colectiva por parte de la sociedad civil para dar respuesta a problemáticas concretas.

\section{Análisis comparativo y conclusiones}

En la tabla 1 se muestra un resumen de los rasgos definitorios que caracterizan a cada uno de los tres modelos descritos en este artículo.

Tabla 1. Resumen de los tres modelos presentados

\begin{tabular}{|c|c|c|c|}
\hline & Influencia en decisiones & Forma jurídica & Tipo de promotor/es \\
\hline $\begin{array}{l}\text { Empresas tradicionales de } \\
\text { economía social y solidaria }\end{array}$ & Basada en la propiedad & $\begin{array}{l}\text { Limitada a unas formas jurídi- } \\
\text { cas definidas. Principalmente } \\
\text { cooperativas y sociedades } \\
\text { laborales }\end{array}$ & Colectivo, sociedad civil \\
\hline B Corporations & $\begin{array}{l}\text { Basada en las relaciones de la } \\
\text { empresa con sus stakeholders }\end{array}$ & Cualquier forma jurídica & Colectivo, sociedad civil \\
\hline Economía del bien común & $\begin{array}{l}\text { Basada en las relaciones de la } \\
\text { empresa con sus stakeholders }\end{array}$ & Cualquier forma jurídica & $\begin{array}{l}\text { Liderada por Christian Felber } \\
\text { en sus inicios, aunque luego se } \\
\text { incorpora más gente }\end{array}$ \\
\hline
\end{tabular}


Colmo se puede ver claramente en la tabla, con respecto a la manera que tiene la sociedad para influir en la toma de decisiones de las empresas, el enfoque tradicional de la economía social y solidaria está basado en el principio clásico de la propiedad del capital, que considera para influir en las empresas hay que formar de la propiedad del capital social. Las $B$ Corp y la economía del bien común modernizan este concepto ampliándolo, puesto que en ambos casos se considera que se puede influir en la toma de decisiones de las empresas tanto si se es propietario como si se tiene un alto grado de interés y de capacidad de influencia, lo que no implica necesariamente formar parte de la propiedad. Por ejemplo, unos consumidores informados y comprometidos pueden influir a través de sus decisiones de compra en el comportamiento de las empresas. De este primer rasgo se deriva el segundo: dado que la propiedad otorga una gran capacidad de influencia en la economía social y solidaria tradicional, se han creado unas formas jurídicas que permitan que parte de la sociedad civil forme parte de la propiedad del capital social. Por su parte, en los otros dos casos las empresas social y medioambientalmente sostenibles y comprometidas pueden tener cualquier forma jurídica.

Finalmente, en cuanto al tipo de promotores de cada una de las iniciativas analizadas, solo en el caso de la economía del bien común nos encontramos con un modelo con un promotor individual que ejerce un liderazgo claro en el proyecto; en los otros dos casos hay promoción colectiva y liderazgos compartidos.

Como principal conclusión podríamos destacar que los nuevos modelos del siglo xxı han contribuido a ampliar y modernizar el ámbito de actuación y el enfoque de la economía social y solidaria clásica, aunque, dado su recorrido histórico, esta está más consolidada y asentada en todo el mundo.

\section{Referencias bibliográficas}

BRYSON, J. M. (2004). «What to do when stakeholders matter: stakeholder identification and analysis techniques». Public Management Review. Vol. 6, n. ${ }^{\circ}$, págs. 21-53.

FREEMAN, R. E. (2010). Strategic management: A stakeholder approach. Cambridge: Cambridge University Press.

JIMÉNEZ, J. C. (coord.) (2005). Un enfoque económico del Tercer Sector. Madrid: Ed. Fundación ONCE.

KAPLAN, R. S; NORTON, D. P. (1996). The balanced scorecard: translating strategy into action. Brighton: Harvard Business Press.

MELIÁN NAVARRO, A.; CAMPOS CLIMENT, V. (2010). «Emprendimiento y economía social como mecanismos de inserción sociolaboral en tiempos de crisis». REVESCO - Revista de Estudios Cooperativos. N. ${ }^{\circ}$ 100, págs. 43-67.

SANCHIS PALACIO, J. R.; CAMPOS CLIMENT, V. (2007). «La Dirección Estratégica en la Economía Social: utilización de herramientas de análisis estratégico en las cooperativas». CIRIEC - España, Revista de Economía Pública, Social y Cooperativa. N. ${ }^{\circ}$ 59, págs. 237-258.

SANCHIS PALACIO, J. R.; CAMPOS CLIMENT, V. (2008). "La innovación social en la empresa: el caso de las cooperativas y de las empresas de economía social en España». Economía Industrial. N. 368, págs. 187-196.

\section{Webgrafía}

https://www.bcorporation.net/ [fecha de consulta: 26 de agosto de 2016].

http://www.cepes.es/ [fecha de consulta: 31 de agosto de 2016].

http://economia-del-bien-comun.org/es [fecha de consulta: 26 de agosto de 2016].

http://www.empleo.gob.es/es/estadisticas/index.htm [fecha de consulta: 31 de agosto de 2016].

http://ica.coop/ [fecha de consulta: 17 de agosto de 2016]. 


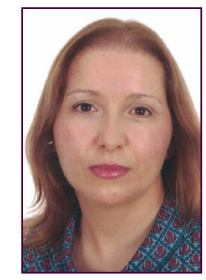

\section{Vanessa Campos-i-Gliment}

vanessa.campos@uv.es

Profesora ayudante doctora del Departamento de Dirección de Empresas.

Universitat de València

Se doctoró en Economía Social (especialidad cooperativas) en 2011, la primera egresada del programa de doctorado en Economía Social de la Universitat de València, pionero en Europa. Hasta el año 2010 ejerció como economista en el sector privado, en el área de consultoría estratégica de empresas. Actualmente es profesora ayudante doctora de la Facultad de Economía de la Universitat de València y ha publicado artículos científicos sobre empresas sociales, cooperativismo y crédito cooperativo desde el año 2001, además de participar en varios proyectos de investigación de ámbito estatal e internacional. Últimamente ha publicado los libros Caixa Popular. Un modelo de banca cooperativa, social y diferente y El modelo Consumo, una cooperativa responsable y sostenible, ambos en Vincle Editorial.

Los textos publicados en esta revista están sujetas -salvo que se indique el contrario- a una licencia de Reconocimiento 3.0 España de Creative Commons. Podéis copiarlos, distribuirlos, comunicarlos públicamente y hacer obras derivadas siempre que reconozcáis los créditos de las obras (autoría, nombre de la revista, institución editora) de la manera especificada por los autores o por la revista. La licencia completa se puede consultar en http://creativecommons.org/licenses/by/3.0/es/deed.ca.

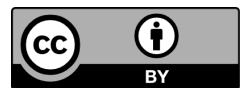

